\title{
RESEÑA. ESCRITORAS EN LENGUA ITALIANA (1880-1920). RENOVACIÓN DEL CANON LITERARIO
}

REVIEW. WRITERS IN ITALIAN LANGUAGE (1880-1920). RENEWAL OF THE LITERARY CANON

Antonella Cagnolati (coord.), Escritoras en lengua italiana (1880-1920). Renovación del canon literario. Granada, Editorial Comares 2019. 
Preceduto da una sintetica introduzione di Antonella Cagnolati, curatrice del volume, il lavoro di ricerca si concentra su alcune figure di indiscusso peso, quali Amalia Guglielminetti, protagonista dei saggi di Mercedes Arriaga Flórez ed Irene Scampuddu; Contessa Lara, con il suo aspetto polimorfico di scrittrice per l'infanzia, al centro dei saggi di Eva Muñoz Raya e Inés Rodríguez; Carolina Invernizio e la sua straordinaria modernità, indagata da Yolanda Romano Martín; la socialista ribelle Anna Franchi, che ha posizioni politiche d'avanguardia sul tema del divorzio, analizzata da Mercedes González de Sande e da Milagro Martín Clavijo; le due grandi esponenti della narrativa, Matilde Serao e Grazia Deledda, oggetto dei saggi rispettivamente di María Reyes Ferrer ed Eva Moreno Lago; l'importante ed eclettica figura di Amalia Pincherle Rosselli, ripercorsa con attenzione da Fabio Contu e José V. Romero; studi di scrittrici il cui nome è oggi quasi dimenticato: Ada Negri, Jolanda, Gina Lombroso, Rosa Rosà, che vengono meritoriamente riportate alla luce da Antonella Cagnolati, Estela González de Sande, María Belén Hernández González, Victoriano Peña Sánchez, oppure legate alla dimensione della letteratura per l'infanzia come Ida Baccini, Emma Perodi, Ida Finzi, rispettivamente analizzate da Salvatore Bartolotta, María Angélica Giordano Paredes e María Dolores Valencia.

Diretto in maniera encomiabile da Antonella Cagnolati, il volume, che è finanziato dall' Assessorato all'Istruzione della Giunta della Castiglia-Leone e dal Fondo Europeo diSviluppo Regionale, scandaglia figure digrandeinteresse della poesia, della narrativa, della letteratura per l'infanzia e della saggistica civile italiana nell'arco di quarant'anni, a cavallo tra ' 800 e '900. Le studiose e gli studiosi, in gran parte docenti o collaboratori in università spagnole, completano i loro saggi con una bibliografia aggiornata degli argomenti da loro trattati. Il libro coglie molto bene l'intreccio osmotico esistente tra le varie autrici, fornendo di ognuna di esse una convincente ed equilibrata chiave di lettura.

Premesso ciò, rendo conto qui di seguito, ancorché in estrema sintesi, dei singoli articoli di questo prezioso e ampio libro che si articola in diciotto capitoli arricchiti da ampie citazioni testuali.

Il saggio di apertura, Las virgenes locas di Amalia Guglielminetti: iconografías rebeldes, è di Mercedes Arriaga Flórez, dell' Ateneo di Siviglia. La studiosa, una delle più insigni italianiste spagnole, sonda con finezza i versi della silloge della Guglielminetti, citando alcuni passi delle poesie da lei stessa tradotte in castigliano. Le sue liriche, come viene notato, sono nel segno della dualità e della contraddizione. Del resto, le protagoniste delle sue poesie passano dall'attesa dell'amore alla cocente disillusione e al disinganno. La poetessa presenta una donna fatale in cui convivono tre emblematiche figure femminili: Medusa, la Sfinge e Litith. Della stessa autrice si occupa nel sedicesimo capitolo Irene Scampuddu, che lavora all'Università di Salamanca. La saggista nelle 
sue pagine, dal titolo La narrativa di Amalia Guglielminetti: Le ore inutili, novelle di donne durante la Grande Guerra, dopo aver ripercorso sinteticamente la produzione della scrittrice, indaga con intelligenza quattro dei dodici racconti pubblicati nel 1919 e raccolti nell'opera citata poco sopra. Imperniate sulla figura del soldato e della donna mostrano in maniera penetrante la società italiana negli anni della prima guerra mondiale, rivelando le emozioni e il vuoto creato da quei tragici eventi.

Il secondo capitolo è costituito da un interessante saggio, Ida Baccini: la voz de la infancia, di cui sono autori Salvatore Bartolotta e María Angélica Giordano Paredes. Dopo aver sottolineato il ruolo unificatore della scuola nell'Italia unita, i due studiosi indagano con acume la figura della Baccini, evidenziando sulla base di una delle sue opere più importanti, Lezioni e racconti per bambini (1882), il suo rilevante contributo pedagogico nella scuola a cavallo tra '800 e 900.

La già ricordata Cagnolati, insigne docente all'Università di Foggia, ha steso il terzo capitolo, "Come una perla di vetro che s'infranga". Cartografie per una metamorfosi nei corpi femminili nella prosa di Ada Negri, che analizza approfonditamente una parte rilevante della produzione della scrittrice, indagando gli effetti devastanti delle violenze subite dalle protagoniste delle sue pagine che ora subiscono mute i colpi inferti al loro corpo e ora reagiscono con la follia e la morte. Segue il saggio, Olga e le altre. Anima di Amelia Pincherle Rosselli in un dialogo intertestuale. Prime note, di Fabio Contu, che ha completato i suoi studi all'Università di Siviglia. Contu scandaglia il primo lavoro teatrale dell'autrice, il cui debutto avvenne nel 1898. Centrata sui motivi dell'onestà intellettuale e della sapiente costruzione psicologica della scrittrice, la pièce è opportunamente messa in rapporto con testi del teatro coevo, da Le vergini di Praga a Diritti dell'anima di Giacosa. Forse senza volerlo, precisa lo studioso, l'autrice crea con Graziana, sorella di Giorgio, che ama la pittrice Olga, un personaggio fresco, capace di far emergere con ironia e sarcasmo l'ipocrisia patriarcale che considera ogni ragazza unicamente una futura moglie e madre.

I due capitoli successivi, El feminismo en la narrativa de Jolanda e Avanti il divorzio, de Anna Franchi: la reividicación femenina a través de la literatura en la Italia del siglo XX, sono di Estela González de Sande e María Mercedes González de Sande, entrambe dell'Università di Oviedo. Il primo saggio approfondisce in maniera mai scontata la figura di Maia Majocchi Plattis, una scrittrice femminista molto prolifica che si segnala non tanto per la netta rivendicazione del diritto della donna ad essere scrittrice, quanto piuttosto per la convinta dichiarazione della sua non inferiorità all'uomo. Il secondo saggio, che è preceduto da alcune acute notazioni sulla condizione delle donne in Italia a cavallo tra XIX e XX secolo, offre una puntigliosa analisi di un testo che evidenzia come la Franchi sia una pioniera nella lotta per l'emancipazione femminile nella quale occupò un ruolo di primo piano. La difesa del divorzio e quella del diritto alla 
procreazione extra-matrimoniale, il riconoscimento legale e sociale di qualsiasi figlio sono condotte con grande determinazione. Della scrittrice si occupa anche in un ampio e articolato saggio Milagro Martín Clavijo, dal titolo En torno a Avanti il divorzio de Anna Franchi. Novela y divorcio a principios del siglo XX. Nelle accurate pagine della studiosa di Salamanca l'argomento è inquadrato nel contesto di quegli anni. Significativamente, il testo della scrittrice, datato 1902, è messo in rapporto con il romanzo Dopo il divorzio (1902) della Deledda, Catene (1882) della Tedeschi Treves e Cavalieri Moderni (1905) della Zampini Salazar, evidenziando che l'opera è una esemplare forma di resistenza contro la violazione dei diritti delle donne.

Segue l'articolo L'anima della donna de Gina Lombroso ne la prensa liberal. In queste pagine, redatte da María Belén Hernández González, dell'Università di Murcia, vengono scandagliate approfonditamente dapprima la vita e l'educazione di una delle figlie di Cesare Lombroso e poi il testo sopra richiamato in cui l'autrice rifiuta la presunta e radicata inferiorità della donna ancorché in maniera non sempre netta. Dall'attenzione sulla stampa spagnola dell'opera anteriore alla sua traduzione si ricava il rilievo delle sue difese dei diritti delle donne. Nel nono capitolo, Personaggi e spazi femminili nella produzione teatrale di Grazia Deledda, Eva María Moreno Lago, dell'Università di Siviglia, firma in un italiano di grande scioltezza, una lucida analisi dei due testi teatrali scritti dall'autrice sarda: Odio vince (1904) e A sinistra. Dopo utili considerazioni sullo spazio scenografico delle due pièces, la studiosa mette in rilievo la forza e l'incisività dei personaggi femminili della Deledda, un'autrice che rivendica la forza e il potere della donna di fronte alla debolezza maschile.

Nel decimo capitolo, El mundo infantil de contessa Lara: desaventuras entre topi y bambole, Eva Muñoz Raya, dell'Università di Granada, scandaglia con ampiezza dapprima Una famiglia di topi (1891), di Evelina Cattermole, più nota col nom de plume Contessa Lara. Il testo indagato trasmette valori quali la uguaglianza e la solidarietà tra gli individui, l'amore per gli animali attraverso la storia di una famiglia di topi inserita in un contesto borghese. La studiosa scandaglia poi Il romanzo della bambola (1895), ponendolo in rapporto con altri suoi testi collegati alla letteratura infantile. Infine, viene studiato il volume Compagni di sventura (1891), in cui animali e uomini sono considerati compagni di viaggio.

Segue un acuto saggio di Victoriano Peña Sánchez, dal titolo Rosa Rosà y el canon del primer futurismo. L'autrice, pseudonimo della viennese Edyth von Haynau, sposatasi nel 1908 con il giornalista italiano Ulrico Amaldi, scelse come forma espressiva proprio l'italiano. Lo studioso dell'Università di Granada, mettendo a frutto le sue ampie conoscenze del Futurismo, si misura con una autrice ancora poco nota, di cui vengono scandagliati i contributi comparsi su "Italia Futurista". La difesa della emancipazione della donna viene espressa con fermezza anche nel romanzo Una donna con tre anime 
(1917). Seguono le pagine di María Reyes Ferrer, dell'Ateneo di Mursia, intitolate Modelos femeninos en la narrativa de Matilde Serao. La saggista scava in profondità nell'eterogenea produzione della Serao, che tratta ora argomenti veristici e ora altri più sentimentali. Le analisi di Piccole anime e poi di Fantasia attestano che l'autrice, come in altri suoi lavori, propone due diversi modelli di donna. Nell'attività giornalistica, ne presenta una appartenente alla piccola borghesia e l'altra alla classe più umile, nella letteratura, pur con qualche eccezione, propone donne sposate per volontà dei genitori e che patiscono il tradimento o l'indifferenza dei mariti e sono, come tali, vittime della società che le condanna all'infelicità.

Nel successivo capitolo, Transgresión y escritura: el caso de Contessa Lara y de Regina de Luanto, Inés Rodriguez Gómez, dell'Università di Valencia, indaga l'attività letteraria e i temi di due autrici, la contessa Lara e Regina di Luanto, alias Guendalina Lipperini Gatti. Quest'ultima stigmatizza l'ipocrisia della società borghese che assegna un ruolo marginale alla donna. In particolare, la saggista, senza peraltro trascurare altri suoi testi, si sofferma sulla sua opera più originale, Salamandra.

Il capitolo quattordicesimo, firmato da Yolanda Romano Martín, dell'Università di Salamanca, dal titolo Carolina Invernizio: el feminismo pasivo de una burguesa de finales del Ottocento, scandaglia con attenzione una scrittrice poco apprezzata da molti scrittori nel periodo fra l'inizio del fascismo e la fine dell'epoca umbertina. Accurata è poi l'analisi de Le operaie italiane. Seguono le pagine di José V. Romero-Rodriguez, Feminismo y feminidad en la obra de Amelia Pincherle Rosselli, ferrea sostenitrice della urgenza per la donna di raggiungere il riconoscimento meritato nella società. La sua ideologia appare evidente nei suoi testi teatrali, da Anima a Illusione a Emma Liona e alla dialettale Il socio di papà. Emma Liona.

Il diciassettesimo capitolo, Emma Perodi e Ida Finzi. Del relato fantástico a la novela de formación, è firmato da María Dolores Valencia Mirón, dell’Università di Granada. Della Perodi, autrice di una produzione vasta e eterogenea, e della Finzi vengono studiate nell'ordine l'opera più conosciuta legata alla letteratura infantile: Le novelle della nonna: fiabe fantastiche (1893) e la interessante narrativa pedagogica.

A chiudere il volume è Sara Velázquez García, dell'Università di Salamanca, con un contributo interessante, La aportación femenina a la construcción de la identidad italiana a través de la literatura infantil y juvenil postrisorgimentale. La saggista sottolinea non solo l'importanza del racconto nella costruzione dell'identità dell'essere umano, ma anche la funzione che riveste nella creazione dell'identità nazionale italiana. Dopo aver parlato dei primi casi del romanzo di formazione all'indomani dell'unificazione italiana, la studiosa precisa l'apporto dato dalle donne all'identità nazionale, contribuendo alla nascita dei futuri cittadini adulti. 
Per concludere, va detto che questa ampia, rigorosa e intelligente mappatura della produzione femminile consente di sondare il rilievo del contributo delle donne nel settore della letteratura e della saggistica. Inoltre, sottolineo che i diciotto saggi di questo ponderoso e importante volume si segnalano per la finezza e la varietà delle letture offerte. 